(C)2009 IEEE. Personal use of this material is permitted. However, permission to reprint/republish this material for advertising or promotional purposes or for creating new collective works for resale or redistribution to servers or lists, or to reuse any copyrighted component of this work in other works must be obtained from the IEEE. 


\title{
Edge Disjoint Paths with Minimum Delay Subject to Reliability Constraint
}

\author{
Ruen Chze Loh, Sieteng Soh, and Mihai Lazarescu \\ Department of Computing \\ Curtin University of Technology, Perth, Australia \\ \{ruen-chze.loh@postgrad.curtin, S.Soh@curtin, M.Lazarescu@curtin\}.edu.au
}

\begin{abstract}
Recently, multipaths solutions have been proposed to improve the quality-of-service (QoS) in communication networks (CN). This paper describes a problem, $\lambda D P / R D$, to obtain the $\lambda$-edge-disjoint-path-set such that its reliability is at least $R$ and its delay is minimal, for $\lambda \geq 1$. $\lambda D P / R D$ is useful for applications that require noncompromised reliability while demanding minimum delay. In this paper we propose an approximate algorithm based on the Lagrange-relaxation to solve the problem. Our solution produces $\lambda D P$ that meets the reliability constraint $R$ with delay $\leq(1+k) D_{\min }$, for $k \geq 1$, and $D_{\min }$ is the minimum path delay of any $\lambda D P$ in the $C N$. Simulations on forty randomly generated CNs show that our polynomial time algorithm produced $\lambda D P$ with delay and reliability comparable to those obtained using the exponential time brute-force approach.
\end{abstract}

Keywords - approximate algorithm; Lagrange relaxation; multi-constrained edge disjoint paths; network reliability; network delay.

\section{INTRODUCTION}

$\mathrm{T}$ HE disjoint path set solutions [1-6] have been proposed to improve the end-to-end quality-of-service $(\mathrm{QoS})$ of the communication networks $(\mathrm{CN})$. Since the number of vertex disjoint paths in general is very limited, the edge disjoint path (DP) set that do not share edges is more commonly used [7]. References [1, 6, 8] propose algorithms to improve the reliability of CNs using DP. Reference [9] also shows that the lifetime of an end-to-end communication can be improved with a higher reliability DP.

Some CNs, such as those for time critical systems and multimedia applications, are subjected to multi-constrained QoS, e.g., reliability, delay, cost and bandwidth. [8] considers cost and delay as the constraint parameters, [10, 11] consider cost and reliability and [4] uses reliability and delay. Note that the problem for generating a DP with two or more constraints has been shown NP-hard [12], and therefore heuristic and approximation algorithms $[8,13,14]$ have been proposed to address the problem.

Orda and Sprintson [13] proposed four approximation algorithms to find two delay-constrained DPs with minimum total cost (2DP/DC). For a $\mathrm{CN}$ that contains two DPs with delay $\leq \mathrm{D}$ and minimal cost OPT, their best algorithm, 2DP4 , always finds $2 \mathrm{DP} / \mathrm{DC}$ with delay $\leq(1+1 / k) \mathrm{D}$ and cost $\leq k(1+\gamma)(1+\varepsilon)$ OPT, where $k$ is a positive integer representing the approximate index, $\gamma$ is a small value bounded by $2(\log k+1) / k$ and $\varepsilon$ is an approximate factor. Applying Lagrange-relaxation, Peng and Shen proposed an algorithm (PSA for short) [8] that improves the performance of $2 \mathrm{DP}-4$ to a delay $\leq(1+1 / k) \mathrm{D}$ with $\cos t \leq(1+k) \mathrm{OPT}$. They showed that PSA can be used to obtain $\lambda \mathrm{DP} / \mathrm{DC}$, for $\lambda>2$. However, both algorithms in [13] and [8] have one significant limitation; they concentrate on finding only 2DP that satisfy the delay and cost constraints whereas other DP may also satisfy the user defined preconditions. In addition, no simulations were performed to benchmark the feasibility of the algorithms and find the optimal value of $k$. Loh, et al [2] have recently described a problem to obtain $\lambda \mathrm{DP} / \mathrm{DR}-$ the set of DPs with maximum reliability subject to delay constraint $D$, for $\lambda \geq 1$. The authors [2] used a similar Lagrange-relaxation method as in [8] to solve this problem.

Our contribution in this paper is twofold. First, we propose an important problem, which is to find $\lambda \mathrm{DP} / \mathrm{RD}-$ the set of DPs with minimum delay subject to reliability $\geq \mathrm{R}$, for $\lambda \geq 1$. The solution to this problem is obviously applicable to some important critical applications, e.g., emergency response, rescue and military operations that demand certain levels of reliability assurances. Such applications require non-compromised reliability while demanding minimum system delay. Second, we present an approximation algorithm to solve this problem. Our solution generates DP with maximum delay no more than $(1+k) D_{\min }$, where $\mathrm{D}_{\min }$ is the minimum delay of a path set in the network.

This paper is organized as follows. Section II discusses the network model, notations and related works as the basis of our approach. Section III formulates the $\lambda \mathrm{DP} / \mathrm{RD}$ problem, while Section IV describes our approximate algorithm. Section $\mathrm{V}$ presents the simulation results and Section VI concludes our paper.

\section{PRELIMINARIES}

\section{A. Network Model and Notations}

A $\mathrm{CN}$ is modeled by an edge-weighted graph $\mathrm{N}=(\mathrm{V}, \mathrm{E}, \mathrm{d}, \mathrm{p})$ where $\mathrm{G}=(\mathrm{V}, \mathrm{E})$ is an undirected graph without multiple edges and self-loops. Each edge $e_{j} \in \mathrm{E}$ is characterized by its delay $d_{j} \in \mathrm{d}$ and its reliability $p_{j} \in \mathrm{p}$, where $d_{j} \geq 0$ is the time taken for traffic to be transferred from one end to the other of $e_{j}$ and $0 \leq p_{j} \leq 1$ represents the probability that $e_{j}$ is UP. An $e_{j}$ is said to be UP (DOWN) if it is functioning (failed). All vertices in $\mathrm{V}$ are assumed to be always UP. The vertices and edges in $\mathrm{N}$ may represent computers and communication links, respectively.

An $(s, t)$ simple path $P_{i}$ between vertices $s$ and $t$ is formed by the set of UP edges such that no vertex is traversed more than once. Any proper subset of a simple path does not result in a path between the vertex pair. The pathset $\mathrm{P}_{s t}$ is a set whose elements are $(s, t)$ simple paths. Fig. 1 shows an 
example network for $s=1$ and $t=11$; the alphabets show the edge names and the values inside each bracket indicate the edge delay and edge reliability respectively. The $\mathrm{P}_{s t}$ of Fig. 1 are: $P_{1}=\{a, b, c, d, e\}, P_{2}=\{a, b, c, r\}, P_{3}=\{a, b, c, h, k, m\}$, $P_{4}=\{f, g, k, m\}, P_{5}=\{f, g, h, d, e\}, P_{6}=\{f, g, h, r\}, P_{7}=\{n, p$, $q, m\}, P_{8}=\{n, p, q, k, h, d, e\}, P_{9}=\{n, p, q, k, h, r\}$.

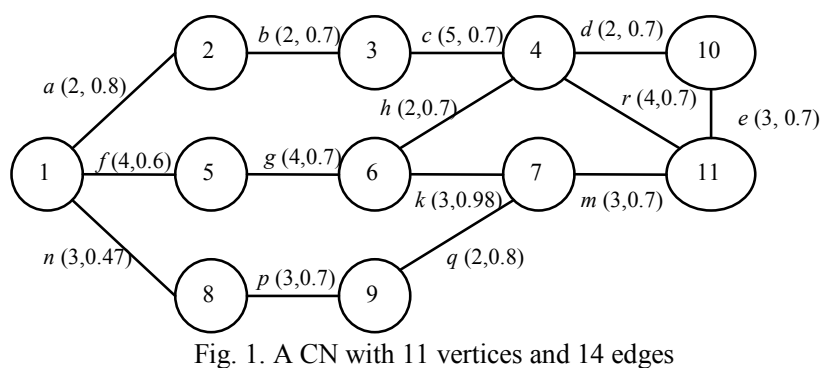

Paths $P_{i}$ and $P_{j}$ are edge disjoint paths (DP) if $e_{\alpha} \neq e_{\beta}$ for each $e_{\alpha} \in P_{i}$ and $e_{\beta} \in P_{j}$. In other words, there is no edge in $P_{i}$ that is in $P_{j}$. Let $\lambda \mathrm{DP}_{\sigma} \subseteq \mathrm{P}_{s t}$ be a DP, where $\lambda \geq 1$ is the total number of paths in the DP, and $\sigma$ is any integer. For a given $\mathrm{P}_{s t}$ there can be more than one $\lambda \mathrm{DP}_{\sigma}$, and none of them is a subset of any other. For example, the $\mathrm{CN}$ in Fig. 1 has six $\lambda \mathrm{DP}_{\sigma}: \quad 3 \mathrm{DP}_{1}=\left\{P_{1}, P_{6}, P_{7}\right\}, \quad 2 \mathrm{DP}_{2}=\left\{P_{1}, P_{9}\right\}, \quad 2 \mathrm{DP}_{3}=\left\{P_{2}, P_{4}\right\}$, $2 \mathrm{DP}_{4}=\left\{P_{2}, P_{8}\right\}, 3 \mathrm{DP}_{5}=\left\{P_{2}, P_{5}, P_{7}\right\}$ and $1 \mathrm{DP}_{6}=\left\{P_{3}\right\}$.

The delay of path $P_{i}, \delta\left(P_{i}\right)$, is the sum of edge delays in $P_{i}$; e.g., $\delta\left(P_{1}\right)=2+2+5+2+3=14$. The delay of $\lambda \mathrm{DP}_{\sigma}, \delta\left(\lambda \mathrm{DP}_{\sigma}\right)$, is the maximum $\delta\left(P_{i}\right)$, for all $P_{i} \in \lambda \mathrm{DP}_{\sigma}$, i.e.,

$$
\delta\left(\lambda \mathrm{DP}_{\sigma}\right)=\max _{P_{i} \in \lambda \mathrm{DP}_{\sigma}}\left(\delta\left(P_{i}\right)\right)
$$

For example, $\delta\left(3 \mathrm{DP}_{1}\right)=\max \quad\left\{\delta\left(P_{1}\right), \delta\left(P_{6}\right), \delta\left(P_{7}\right)\right\}=$ $\max \{14,14,11\}=14$.

The $(s, t)$ reliability, $\rho\left(P_{i}\right)$, of a simple path $P_{i}$, is computed by multiplying $p_{j}$ of each $e_{j}$ that forms $P_{i}$, i.e.,

$$
\rho\left(P_{i}\right)=\prod_{e_{j} \in P_{i}} p_{j}
$$

For example, $\rho\left(P_{1}\right)=0.8 * 0.7^{4}=0.19208$. The disjoint paths in an $\lambda \mathrm{DP}_{\sigma}$ can be viewed as the components of a parallel system [15], and therefore its reliability, $\rho\left(\lambda \mathrm{DP}_{\sigma}\right)$, can be computed as:

$$
\rho\left(\lambda \mathrm{DP}_{\sigma}\right)=1-\prod_{P_{i} \in \lambda \mathrm{DP}_{\sigma}}\left(1-\rho\left(P_{i}\right)\right)
$$

The multiplicative operations in (3) can be transformed into additive operations as:

$$
\rho\left(\lambda \mathrm{DP}_{\sigma}\right)=1-\log ^{-1}\left(\sum_{P_{i} \in \lambda \mathrm{DP}_{\sigma}} \log \left(1-\rho\left(P_{i}\right)\right)\right),
$$

where $\log ^{-1}(\mathrm{X})$ is the antilog of $\mathrm{X}$. Using Eq. (3), $\rho\left(3 \mathrm{DP}_{1}\right)=$ $\left(1-\left(1-\rho\left(P_{1}\right)\right) *\left(1-\rho\left(P_{6}\right)\right) *\left(1-\rho\left(P_{7}\right)\right)\right)=1-\left(\left(1-0.8 * 0.7^{4}\right) *(1-\right.$ $\left.\left.0.6 * 0.7^{3}\right) *\left(1-0.47 * 0.8 * 0.7^{2}\right)\right)=0.4766$. Note, $\rho\left(3 D_{1}\right)$ can be equivalently computed using Eq. (4) as $1-\log ^{-1}((-$ $0.0926)+(-0.1001)+(-0.0884))=1-\log ^{-1}(-0.2690)=0.4766$.

\section{B. Related Work}

The Peng and Shen algorithm (PSA) [8] utilizes the Lagrange-relaxation to approximately generate 2DP/DC from a graph $\mathrm{N}(\mathrm{V}, \mathrm{E}, \mathrm{d}, \mathrm{c})$, where $\mathrm{c}$ is the set of edge cost and $d$ is the set of edge delay in $G(V, E)$. For a given delay constraint $\mathrm{D}$, PSA produces a $2 \mathrm{DP} / \mathrm{DC}$ with delay less than $(1+1 / k) \mathrm{D}$ and a total cost no more than $(1+k) \mathrm{OPT}$, where OPT is the optimal cost among all 2DP that meet the delay constraint. The total cost of the 2DP/DC is defined as the sum of the cost of each path $P_{i} \in 2 \mathrm{DP}_{\sigma}$. Note that the algorithm can be extended to produce $\lambda \mathrm{DP} / \mathrm{DC}$, for $\lambda>2$ [8]. Here, PSA aims to generate the minimum cost $\lambda \mathrm{DP} / \mathrm{DC}$ with the largest $\lambda$ that meets the delay constraint. In contrast, a $\lambda \mathrm{DP} / \mathrm{RD}$ is not necessarily a DP with the largest $\lambda$. Therefore, PSA is not suitable for generating $\lambda \mathrm{DP} / \mathrm{RD}$.

The algorithm in [2] uses a similar technique to PSA to produce a $\lambda \mathrm{DP} / \mathrm{DR}$ for a given delay constraint $\mathrm{D}$ and a graph $\mathrm{N}(\mathrm{V}, \mathrm{E}, \mathrm{d}, \mathrm{p})$ where $\mathrm{p}$ is the set of edge reliability and $\mathrm{d}$ is the set of edge delay in $G(V, E)$. The algorithm [2] is guaranteed to produce a $\lambda \mathrm{DP}$ with, respectively, delay and reliability bounded by Eq. (5) and Eq. (6):

$$
\begin{gathered}
\delta(\lambda \mathrm{DP}) \leq(1+1 / k) \mathrm{D} \\
|\log (1-\rho(\lambda \mathrm{DP}))| \leq(1+k)|\log (1-\mathrm{OPT})|,
\end{gathered}
$$

where OPT is the maximum reliability among all possible $\lambda \mathrm{DP}$ that satisfy the delay requirement, D. Like $\lambda \mathrm{DP} / \mathrm{RD}$, the generated $\lambda \mathrm{DP} / \mathrm{DR}$ is not necessarily a DP with the maximum $\lambda$. One may use the algorithm in [2] to generate $\lambda \mathrm{DP} / \mathrm{RD}$ if one substitutes D in Eq. (5), and OPT in Eq. (6) with the maximum path delay $\mathrm{D}_{\max }$ of the network and the reliability requirement $\mathrm{R}$, respectively. Notice that the generated $\lambda \mathrm{DP} / \mathrm{RD}$ would have a reliability value at most $(1+k)^{*}|\log (1-\mathrm{R})|$, which may not satisfy the reliability requirement, $\mathrm{R}$. Therefore, a more effective algorithm, presented in this paper, is needed to solve the $\lambda \mathrm{DP} / \mathrm{RD}$ problem.

\section{III. $\lambda \mathrm{DP} / \mathrm{RD}$ PROBLEM FORMULATION}

For a given reliability constraint $\mathrm{R}$, let $\lambda \mathrm{DP}_{\sigma}{ }^{r}$ be $\lambda \mathrm{DP}_{\sigma}$ that has reliability at least R, i.e., $\rho\left(\lambda \mathrm{DP}_{\sigma}{ }^{r}\right) \geq \mathrm{R}$. Let $\lambda \mathrm{DP}_{\tau}^{r d}$ be $\lambda \mathrm{DP}_{\sigma}^{r}$ with minimum delay, i.e.,

$$
\delta\left(\lambda \mathrm{DP}_{\tau}^{r d}\right)=\min \left(\left\{\delta\left(\lambda \mathrm{DP}_{\sigma}^{r}\right)\right\}\right)
$$

Note that there can be more than one $\lambda \mathrm{DP}_{\sigma}^{r d}$. The edgedisjoint-path-set with minimum delay and reliability $\geq \mathrm{R}$ problem $(\lambda \mathrm{DP} / \mathrm{RD})$ is to find among all $\lambda \mathrm{DP}_{\tau}^{\text {rd }}$ a DP with the highest reliability (called $\lambda \mathrm{DP}^{\mathrm{BM}}$ ), i.e.,

$$
\rho\left(\lambda \mathrm{DP}^{\mathrm{BM}}\right)=\max \left(\left\{\rho\left(\lambda \mathrm{DP}_{\tau}^{r d}\right)\right\}\right)
$$

To illustrate the $\lambda \mathrm{DP} / \mathrm{RD}$ problem, consider $\mathrm{R}=0.4$ and the $\lambda \mathrm{DP}_{\sigma} \mathrm{s}$ of Fig. 1. Using Eq. (4), we obtained, $\rho\left(3 \mathrm{DP}_{1}\right)=0.4766, \quad \rho\left(2 \mathrm{DP}_{2}\right)=0.2942, \quad \rho\left(2 \mathrm{DP}_{3}\right)=0.4836$, $\rho\left(2 \mathrm{DP}_{4}\right)=0.3386, \quad \rho\left(3 \mathrm{DP}_{5}\right)=0.4934$ and $\rho\left(1 \mathrm{DP}_{6}\right)=0.1882$. Among the $\lambda \mathrm{DP}_{\sigma} \mathrm{s}$ there are three $\lambda \mathrm{DP}_{\sigma}{ }^{r}: 3 \mathrm{DP}_{1}{ }^{\mathrm{r}}, 2 \mathrm{DP}_{3}{ }^{\mathrm{r}}$ and $3 \mathrm{DP}_{5}{ }^{\mathrm{r}}$. Using Eq. (1), we obtain, $\delta\left(3 \mathrm{DP}_{1}{ }^{\mathrm{r}}\right)=14, \delta\left(2 \mathrm{DP}_{3}{ }^{\mathrm{r}}\right)=14$ and $\delta\left(3 \mathrm{DP}_{5}{ }^{\mathrm{r}}\right)=15$; thus using Eq. (7), there are two $\lambda \mathrm{DP}_{\sigma}{ }^{r d}$ : $3 \mathrm{DP}_{1}{ }^{\mathrm{r}}, 2 \mathrm{DP}_{3}{ }^{\mathrm{r}}$. Eq. (8) obtains $\max \left\{\rho\left(3 \mathrm{DP}_{1}{ }^{\mathrm{r}}\right)=0.4766\right.$, $\left.\rho\left(2 \mathrm{DP}_{3}{ }^{\mathrm{r}}\right)=0.4836\right\}=0.4836$, and thus $\lambda \mathrm{DP}^{\mathrm{BM}}=2 \mathrm{DP}_{3}$. Notice that the optimal $2 \mathrm{DP}_{3}$ is not a $\lambda \mathrm{DP}$ with the largest $\lambda$.

One may obtain $\lambda \mathrm{DP}^{\mathrm{BM}}$ by exhaustively generating all possible $\lambda \mathrm{DP}_{\sigma}{ }^{r}$ path sets and using Eq. (7) to select the set of $\lambda \mathrm{DP}_{\sigma}{ }^{r d}$, and using Eq. (8) select the most reliable one. Note that $\mathrm{N}(\mathrm{V}, \mathrm{E}, \mathrm{d}, \mathrm{p})$, in general, contains an exponential number (in terms of $|\mathrm{E}|$ ) of $(s, t)$ paths $\left(\left|\mathrm{P}_{s t}\right|\right)$, and therefore this brute force $(\mathrm{BF})$ approach may generate an exponential number (in terms of $\left.\left|\mathrm{P}_{s t}\right|\right)$ of $\lambda \mathrm{DP}_{\sigma}{ }^{r}$, and thus this solution has double exponential (in terms of $|\mathrm{E}|$ ) time complexity. 


\section{THE $\lambda$ DP/RD APPROXIMATE ALGORITHM}

\section{A. Lagrange-relaxation Approach for $\lambda D P / R D$}

We propose to use Lagrange-relaxation technique to solve the $\lambda \mathrm{DP} / \mathrm{RD}$ problem. As in [8] and [2], we combine the reliability $p_{i}$ and delay $d_{i}$ of each edge $e_{i}$ into a weight $w_{i}=$ $d_{i}+\eta^{*} \log \left(p_{i}\right)$, rather than considering them separately. In other words, we transform the two constraint-weighted network $\mathrm{N}(\mathrm{V}, \mathrm{E}, \mathrm{p}, \mathrm{d})$ into the one-constraint-weighted network N(V,E,w), for $w_{i} \in \mathrm{w}$. The value of $\eta$ should be set properly to minimize the delay $\delta(\lambda \mathrm{DP})$, and to maximize reliability $\rho(\lambda D P) \geq R$. Note that for $\lambda \mathrm{DP} / \mathrm{DC}$, it had been shown that setting $\eta$ (called $\alpha$ in [8]) to $k^{*} \mathrm{OPT} / \mathrm{D}$ produced good results. Since $\lambda \mathrm{DP} / \mathrm{RD}$ is similar to $\lambda \mathrm{DP} / \mathrm{DC}$, we set $\eta_{k}=k^{*} \mathrm{D}_{\min } / \log (\mathrm{R})$, i.e., replacing $\mathrm{OPT}$ with $\mathrm{D}_{\min }$ and the delay constraint $\mathrm{D}$ with the $\log$ of the reliability constraint $R$, where $D_{\text {min }}$ is the minimum delay of all possible DPs in the network. One may obtain $\mathrm{D}_{\text {min }}$ by using DPSP [9] or iDPSP [6] from $\mathrm{N}(\mathrm{V}, \mathrm{E}, \mathrm{p}, \mathrm{d})$ assuming perfect edges, i.e., $p_{i}=1$ for all $p_{i} \in \mathrm{p}$. The following lemma states that when the value of $|\eta|$ increases, $\rho(\lambda \mathrm{DP})$ decreases.

Let $\mathrm{W}\left(\lambda \mathrm{DP}_{\sigma}, \eta_{k}\right)=\delta\left(\lambda \mathrm{DP}_{\sigma}\right)+\eta_{k} * \log \left(\rho\left(\lambda \mathrm{DP}_{\sigma}\right)\right)$ denote the weight of $\lambda \mathrm{DP}_{\sigma}$ generated when $\eta=\eta_{k}$. In graph $\mathrm{G}=(\mathrm{V}, \mathrm{E})$, let $\lambda \mathrm{DP}^{\alpha}$ be the DP with the minimum total weight when we set $w_{i}=d_{i}+\eta_{\alpha}{ }^{*} \log \left(p_{i}\right)$ and $\lambda \mathrm{DP}^{\beta}$ be the DP with the minimum total weight when we set $w_{i}=d_{i}+\eta_{\beta}{ }^{*} \log \left(p_{i}\right)$, i.e., for $k=\alpha$ and $k=\beta$, respectively.

Lemma 1. $\log \left(\rho\left(\lambda D P^{\beta}\right)\right) \leq \log \left(\rho\left(\lambda D P^{\alpha}\right)\right)$, for $0<\left|\eta_{\alpha}\right| \leq\left|\eta_{\beta}\right|$. Proof: Since $\lambda \mathrm{DP}^{\alpha}$ is the DP with the minimum total weight when we set $w_{i}=d_{i}+\eta_{\alpha}{ }^{*} \log \left(p_{i}\right)$, we have,

$$
\begin{gathered}
\mathrm{W}\left(\lambda \mathrm{DP}^{\alpha}, \eta_{\alpha}\right)=\delta\left(\lambda \mathrm{DP}^{\alpha}\right)+\eta_{\alpha}{ }^{*} \log \left(\rho\left(\lambda \mathrm{DP}^{\alpha}\right)\right) \\
\leq \delta\left(\lambda \mathrm{DP}^{\beta}\right)+\eta_{\alpha}{ }^{*} \log \left(\rho\left(\lambda \mathrm{DP}^{\beta}\right)\right)
\end{gathered}
$$

Since $\lambda \mathrm{DP}^{\beta}$ is the DP with the minimum total weight when we set $w_{i}=d_{i}+\eta_{\beta}{ }^{*} \log \left(p_{i}\right)$, we have,

$$
\begin{gathered}
\mathrm{W}\left(\lambda \mathrm{DP}^{\beta}, \eta_{\beta}\right)=\delta\left(\lambda \mathrm{DP}^{\beta}\right)+\eta_{\beta}{ }^{*} \log \left(\rho\left(\lambda \mathrm{DP}^{\beta}\right)\right) \\
\leq \delta\left(\lambda \mathrm{DP}^{\alpha}\right)+\eta_{\beta}{ }^{*} \log \left(\rho\left(\lambda \mathrm{DP}^{\alpha}\right)\right)
\end{gathered}
$$

Adding Eq. (9) and Eq. (10), we obtain:

$\delta\left(\lambda \mathrm{DP}^{\alpha}\right)+\eta_{\alpha}{ }^{*} \log \left(\rho\left(\lambda \mathrm{DP}^{\alpha}\right)\right)+\delta\left(\lambda \mathrm{DP}^{\beta}\right)+\eta_{\beta} * \log \left(\rho\left(\lambda \mathrm{DP}^{\beta}\right)\right)$ $\leq \delta\left(\lambda \mathrm{DP}^{\beta}\right)+\eta_{\alpha}{ }^{*} \log \left(\rho\left(\lambda \mathrm{DP}^{\beta}\right)\right)+\delta\left(\lambda \mathrm{DP}^{\alpha}\right)+\eta_{\beta}{ }^{*} \log \left(\rho\left(\lambda \mathrm{DP}^{\alpha}\right)\right)$, and thus $\log \left(\rho\left(\lambda \mathrm{DP}^{\beta}\right)\right) \leq \log \left(\rho\left(\lambda \mathrm{DP}^{\alpha}\right)\right)$.

Theorem 1. $\rho\left(\lambda \mathrm{DP}^{\beta}\right) \leq \rho\left(\lambda \mathrm{DP}^{\alpha}\right)$, for $1 \leq \alpha \leq \beta$.

Proof: By definition, $\eta_{\beta}=\beta * D_{\min } / \log (\mathrm{R}) \quad$ and $\eta_{\alpha}=\alpha^{*} D_{\min } / \log (R)$, and therefore, $\left|\eta_{\alpha}\right| \leq\left|\eta_{\beta}\right|$. Since $0 \leq \rho\left(\lambda D P^{\beta}\right)$ $\leq 1$ and $0 \leq \rho\left(\lambda D P^{\alpha}\right) \leq 1$, by Lemma $1 \rho\left(\lambda \mathrm{DP}^{\beta}\right) \leq \rho\left(\lambda \mathrm{DP}^{\alpha}\right)$ for $\left|\eta_{\alpha}\right| \leq\left|\eta_{\beta}\right|$, and thus for $1 \leq \alpha \leq \beta$.

Theorem 2. Consider a $\lambda \mathrm{DP}^{k}$ that is obtained when $\eta_{k}=k^{*} \mathrm{D}_{\min } / \log (\mathrm{R}), \quad \mathrm{D}_{\min } \leq \mathrm{W}\left(\lambda \mathrm{DP}^{k}, \eta_{k}\right) \leq(1+2 k) \mathrm{D}_{\min } \quad$ iff $\mathrm{D}_{\text {min }} \leq \delta\left(\lambda \mathrm{DP}^{k}\right) \leq(1+k) \mathrm{D}_{\text {min }}$.

Proof: To prove that $\mathrm{D}_{\min } \leq \mathrm{W}\left(\lambda \mathrm{DP}^{k}, \eta_{k}\right) \leq(1+2 k) \mathrm{D}_{\min }$ if $\mathrm{D}_{\text {min }} \leq \delta\left(\lambda \mathrm{DP}^{k}\right) \leq(1+k) \mathrm{D}_{\text {min }}$, consider Fig. 2 , which describes the feasible solution space for $\lambda \mathrm{DP} / \mathrm{RD}$. The $\mathrm{x}$-axis represents the logarithm of all possible reliability values $\rho(\lambda \mathrm{DP})$ when $\delta(\lambda \mathrm{DP})=0$, i.e., when each edge has zero delay. On the other hand, the $y$-axis represents all possible delay values $\delta(\lambda \mathrm{DP})$ assuming perfect edges, i.e., $\rho(\lambda \mathrm{DP})=1$ or $\log (\rho(\lambda \mathrm{DP})=0$. Since the minimum delay (lower bound) of all possible DPs in the network is $D_{\min }$, the delay values of any point in line $A B$ is $D_{\text {min }}$. A feasible solution must have a reliability of at least $R$, and therefore the value of each point in line $B D$ is $\log (\mathrm{R})$. Since the algorithm aims to obtain a $\lambda \mathrm{DP}$ with delay at most $(1+k) \mathrm{D}_{\min }$, line $\mathrm{CD}$ represents the upper bound of $\delta(\lambda \mathrm{DP})$. Therefore, Fig. 2 shows $\mathrm{D}_{\min } \leq \delta\left(\lambda \mathrm{DP}^{k}\right) \leq(1+k) \mathrm{D}_{\min }$ and $\log \left(\rho\left(\lambda \mathrm{DP}^{k}\right)\right) \geq \log (\mathrm{R})$.

The upper and lower bounds of the weight of $\lambda \mathrm{DP}^{k}$, $\mathrm{W}\left(\lambda \mathrm{DP}^{k}, \eta_{k}\right)$, can be obtained as follows. Substituting $\log \left(\rho\left(\lambda \mathrm{DP}^{k}\right)=0 \quad\right.$ into $\quad \mathrm{W}\left(\lambda \mathrm{DP}^{k}, \eta_{k}\right) \quad=\delta\left(\lambda \mathrm{DP}^{k}\right)+$ $\eta_{k}{ }^{*} \log \left(\rho\left(\lambda \mathrm{DP}^{k}\right)\right)$, the weights of a $\lambda \mathrm{DP}^{k}$ at points $\mathrm{A}$ and $\mathrm{C}$ are $\mathrm{D}_{\text {min }}$ and $(1+k) \mathrm{D}_{\text {min }}$, respectively. Note that $\delta\left(\lambda \mathrm{DP}^{k}\right)=\mathrm{D}_{\text {min }}$ at point $\mathrm{A}$, and $\delta\left(\lambda \mathrm{DP}^{k}\right)=(1+k) \mathrm{D}_{\min }$ at point $\mathrm{C}$. Similarly, substituting $\rho\left(\lambda \mathrm{DP}^{k}\right)=\mathrm{R}$ and $\eta_{k}=k^{*} \mathrm{D}_{\min } / \log (\mathrm{R})$ into $\mathrm{W}\left(\lambda \mathrm{DP}^{k}, \eta_{k}\right)=\delta\left(\lambda \mathrm{DP}^{k}\right)+\eta_{k}^{*} \log \left(\rho\left(\lambda \mathrm{DP}^{k}\right)\right)$, we obtain $\mathrm{W}\left(\lambda \mathrm{DP}^{k}, \eta_{k}\right)=\delta\left(\lambda \mathrm{DP}^{k}\right)+k^{*} \mathrm{D}_{\text {min }}$, and since $\delta\left(\lambda \mathrm{DP}^{k}\right)=\mathrm{D}_{\text {min }}$ at $\mathrm{B}$ and $\delta\left(\lambda \mathrm{DP}^{k}\right)=(1+k) \mathrm{D}_{\min }$ at $\mathrm{D}$, the weights at $\mathrm{B}$ and $\mathrm{D}$ are $(1+k) \mathrm{D}_{\min }$ and $(1+2 k) \mathrm{D}_{\min }, \quad$ respectively. Therefore, $\mathrm{D}_{\min } \leq \mathrm{W}\left(\lambda \mathrm{DP}^{k}, \eta_{k}\right) \leq(1+2 k) \mathrm{D}_{\min }$ if $\mathrm{D}_{\min } \leq \delta\left(\lambda \mathrm{DP}^{k}\right) \leq(1+k) \mathrm{D}_{\min }$

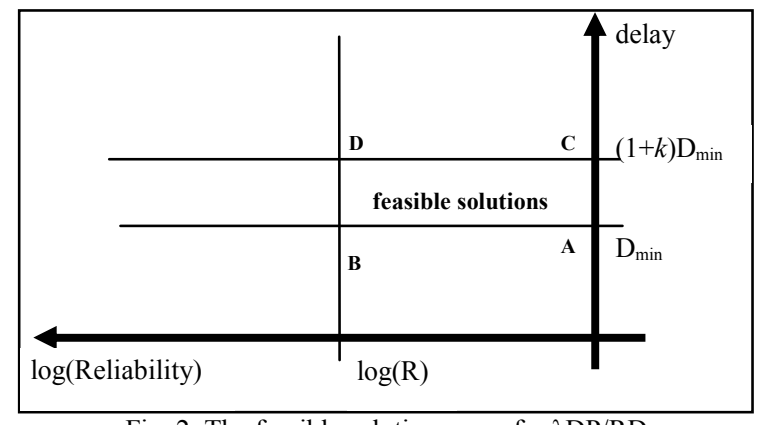

Fig. 2. The feasible solution space for $\lambda \mathrm{DP} / \mathrm{RD}$

To prove that $\mathrm{D}_{\text {min }} \leq \delta\left(\lambda D \mathrm{P}^{k}\right) \leq(1+k) \mathrm{D}_{\text {min }}$ if $\mathrm{D}_{\text {min }} \leq$ $\mathrm{W}\left(\lambda \mathrm{DP}^{k}, \eta_{k}\right) \leq(1+2 k) \mathrm{D}_{\min }$, one may notice that any $\lambda \mathrm{DP}$ that is generated when its weight is at a point $(x, y)$ in the feasible solution, shown in Fig. 2, will give a delay of $y$. The maximum weight $\mathrm{W}\left(\lambda \mathrm{DP}^{k}, \eta_{k}\right)=(1+2 k) \mathrm{D}_{\min }$ is obtained when the point is at $\mathrm{D}$, i.e., when $\log \left(\rho\left(\lambda \mathrm{DP}^{k}\right)\right)=\log (\mathrm{R})$. Thus, at that point, $\mathrm{W}\left(\lambda \mathrm{DP}^{k}, \eta_{k}\right)=\delta\left(\lambda \mathrm{DP}^{k}\right)+k^{*} \mathrm{D}_{\text {min }} * \log (\mathrm{R}) / \log (\mathrm{R})$ $\leq(1+2 k) \mathrm{D}_{\min }$ or $\delta\left(\lambda \mathrm{DP}^{k}\right) \leq(1+2 k) \mathrm{D}_{\text {min }}-k^{*} \mathrm{D}_{\text {min }}=(1+k) \mathrm{D}_{\text {min }}$. Therefore, $\delta\left(\lambda \mathrm{DP}^{k}\right) \leq(1+k) \mathrm{D}_{\text {min }}$. Similarly, the minimum weight $\mathrm{W}\left(\lambda \mathrm{DP}^{k}, \eta_{k}\right)=\mathrm{D}_{\min }$ is generated at point $\left(0, \mathrm{D}_{\min }\right)$, which is at $\mathrm{A}$, where $\log \left(\rho\left(\lambda \mathrm{DP}^{k}\right)\right)=0$. Thus, at this point, $\mathrm{W}\left(\lambda \mathrm{DP}^{k}, \eta_{k}\right)=\delta\left(\lambda \mathrm{DP}^{k}\right)+k^{*} \mathrm{D}_{\min } * 0 / \log (\mathrm{R}) \geq \mathrm{D}_{\min }$ or $\delta\left(\lambda \mathrm{DP}^{\mathrm{k}}\right)$ $\geq \mathrm{D}_{\min }$.

\section{B. Algorithm}

Fig. 3 shows the $\lambda \mathrm{DP} / \mathrm{RD}$ algorithm. Let $\lambda \mathrm{DP}^{\mathrm{Rmax}}$ be the $\lambda \mathrm{DP}$ with the maximum reliability, and $\lambda \mathrm{DP}^{\mathrm{Dmin}}$ be the $\lambda \mathrm{DP}$ with the minimum total delay in $\mathrm{N}=(\mathrm{V}, \mathrm{E}, \mathrm{p}, \mathrm{d})$. One may use the DPSP [9] or the iDPSP [6] algorithm to compute both $\lambda \mathrm{DP}^{\mathrm{Rmax}}$ and $\lambda \mathrm{DP}^{\mathrm{Dmin}}$ from N(V,E,d,p) by setting each $d_{i} \in \mathrm{d}$ to 0 and $p_{i} \in \mathrm{p}$ to 1 , respectively. If the reliability of $\lambda \mathrm{DP}^{\mathrm{Rmax}}$ is less than the reliability constraint $\mathrm{R}$, there is no feasible solution for the network, and therefore the algorithm terminates. Otherwise, the algorithm aims to obtain the optimal $\lambda \mathrm{DP}^{\mathrm{BM}}$ that has best metrics $\mathrm{BM}=$ (delay $_{\mathrm{BM}}$, reliability $\left.{ }_{\mathrm{BM}}\right) ; \lambda \mathrm{DP}^{\mathrm{BM}}$ and $\mathrm{BM}$ are initialized to $\varnothing$ set and $(\infty$, $0)$, respectively. Each iteration in the loop uses $\operatorname{iDPSP}()$ to obtain a new $\lambda \mathrm{DP}, \lambda \mathrm{DP}^{\mathrm{NM}}$, that has the minimum weight from $\mathrm{N}(\mathrm{V}, \mathrm{E}, \mathrm{w})$, where the edge weights, $w_{i} \in \mathrm{w}$ are calculated for each increasing $k$. Note that $\lambda \mathrm{DP}^{\mathrm{NM}}$ has new metrics $\mathrm{NM}=\left(\right.$ delay $_{\mathrm{NM}}$, reliability $\left.{ }_{\mathrm{NM}}\right)$. Following Theorem 1 , if $\rho\left(\lambda D P^{\mathrm{NM}}\right)<\mathrm{R}$ is generated when $k=\alpha$, then all other $\rho\left(\lambda \mathrm{DP}^{\mathrm{NM}}\right)$ generated when $k=\beta$ will always be less than $\mathrm{R}$ 
for $\beta>\alpha$. Thus, the algorithm stops the iteration and return $\lambda \mathrm{DP}^{\mathrm{BM}}$ as the approximately best $\lambda \mathrm{DP}$. Otherwise, from Theorem 2, if $\mathrm{D}_{\text {min }} \leq \mathrm{W}\left(\lambda \mathrm{DP}^{k}, \eta_{k}\right) \leq(1+2 k) \mathrm{D}_{\text {min }}$, we use function optimal() to select the more optimal $\lambda \mathrm{DP}$ between $\lambda \mathrm{DP}^{\mathrm{BM}}$ and $\lambda \mathrm{DP}^{\mathrm{NM}}$, and continue the iteration for larger $k$. From Eqs. (7) and (8), $\lambda \mathrm{DP}^{\mathrm{BM}}$ is more optimal than $\lambda \mathrm{DP}^{\mathrm{NM}}$ if (i) delay $_{\mathrm{BM}}<$ delay $_{\mathrm{NM}}$, or (ii) delay $_{\mathrm{BM}}=$ delay $_{\mathrm{NM}}$ and reliability $_{\mathrm{BM}}>$ reliability $_{\mathrm{NM}}$.

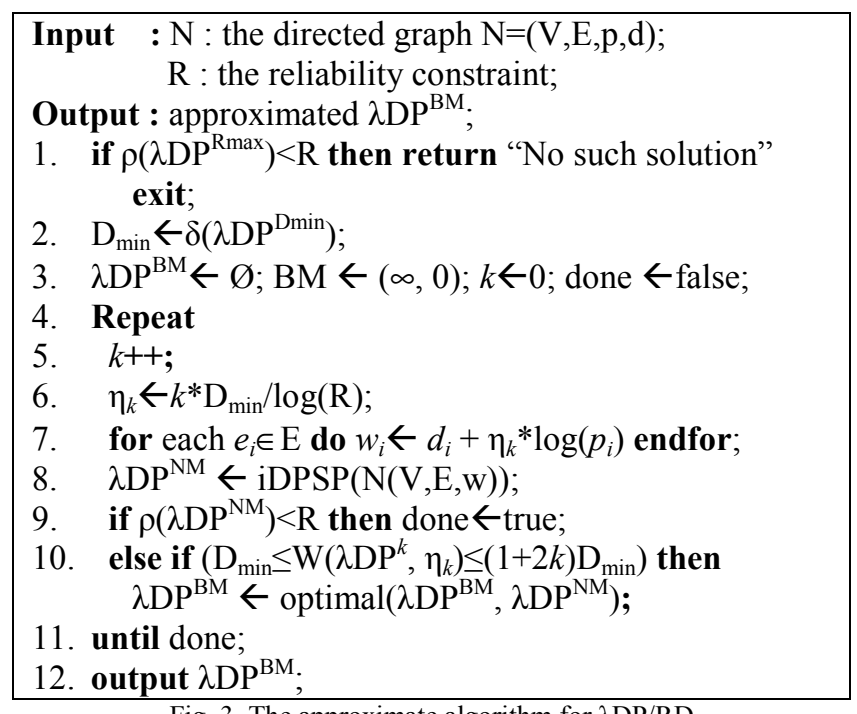
Fig. 3. The approximate algorithm for $\lambda \mathrm{DP} / \mathrm{RD}$

The time complexity of the algorithm can be calculated as follows. Either DPSP [9] or iDPSP [6] have the time complexity of $\mathrm{O}\left(|\mathrm{V} \| \mathrm{E}|^{2}\right)$, and line 7 and optimal() has $\mathrm{O}(\mathrm{E})$ and $\mathrm{O}(1)$ time complexity, respectively. Therefore, the time complexity of the algorithm depends on the total number the loop is repeated, i.e., on the value of $k$. Thus, time complexity is $\mathrm{O}\left(k^{*}|\mathrm{~V}||\mathrm{E}|^{2}\right)$.

As an illustrating example, consider the network in Fig. 1 with $\mathrm{R}=0.4$. Using iDPSP [6], we obtained $\rho\left(\lambda \mathrm{DP}^{\mathrm{Rmax}}\right)=0.4934$ and $\delta\left(\lambda \mathrm{DP}^{\mathrm{Dmin}}\right)=11$. Table I shows the delay, reliability and weight of the $\lambda \mathrm{DP}$ obtained by the algorithm when $k$ was incremented from 1 to 3 .

TABLE I

RESULTS OBTAINED BY THE ALGORITHM FOR CN IN FIG.1

\begin{tabular}{|c|c|c|c|}
\hline $\boldsymbol{k}$ & Delay & Reliability & $\mathbf{W}\left(\boldsymbol{\lambda} \mathbf{D P}^{\boldsymbol{k}}, \boldsymbol{\eta}_{\boldsymbol{k}}\right)$ \\
\hline 1 & 15 & 0.493437 & 15.7 \\
\hline 2 & 14 & 0.483619 & 27.5 \\
\hline 3 & 18 & 0.338637 & 39.3 \\
\hline
\end{tabular}

When $\quad k=1, \quad \mathrm{~W}\left(\lambda \mathrm{DP}^{k}, \eta_{k}\right)=15.7 \quad$ which satisfies $11 \leq \mathrm{W}\left(\lambda \mathrm{DP}^{1}, \eta_{1}\right) \leq 33$ thus, $\mathrm{BM}=(15,0.493437)$. When $k$ was increased to $2, \mathrm{~W}\left(\lambda \mathrm{DP}^{2}, \eta_{2}\right)=27.5$ which still satisfies $11 \leq \mathrm{W}\left(\lambda \mathrm{DP}^{2}, \eta_{2}\right) \leq 55$ and the delay ${ }_{\mathrm{NM}}$ obtained was 14 which was lesser than delay ${ }_{\mathrm{BM}}$, therefore, line 10 replaces $\lambda \mathrm{DP}^{\mathrm{BM}}$ with $\lambda \mathrm{DP}^{\mathrm{NM}}$ with $\mathrm{BM}=(14,0.483619)$. For $k=3$, the reliability $\mathrm{NM}_{\mathrm{N}}<\mathrm{R}$ so the algorithm output the $\lambda \mathrm{DP}^{\mathrm{BM}}=\left\{P_{2}, P_{4}\right\}$ obtained when $k=2$ with $\mathrm{BM}=(14,0.483619)$.

\section{Simulation AND DISCUSSION}

We used BRITE [16] with the RTWaxman configuration to generate a random topology that contains 50 vertices, 72 edges and $1124 s$ - $t$ paths. From the topology, we constructed 40 random networks, 10 each for the following four different network groups, CN1, CN2, CN3, and CN4. The edges of each network in $\mathrm{CN} 1$ and $\mathrm{CN} 3$ are randomly assigned with edge reliabilities ranging from 0.1 to 0.5 and
0.1 to 0.9 respectively with incremental value of 0.1 . For $\mathrm{CN} 2$, we used edge reliability values ranging from 0.5 to 0.75 with incremental value of 0.05 , while for $\mathrm{CN} 4$ the random reliability values are ranging from 0.9 to 0.99 with incremental values of 0.01 . Further, we also assigned a random delay value ranging from 3 to 7 units to each edge of the 40 networks, and used reliability constraints $\mathrm{R}$ of 0.1 , 0.5, 0.7 and 0.95 for $\mathrm{CN} 1, \mathrm{CN} 2, \mathrm{CN} 3$, and $\mathrm{CN} 4$, respectively. Note that we generated the 40 random networks such that each of them satisfies the constraint $\mathrm{R}$. In all our simulations, the value of $k$ starts from 1 . We used the $\mathrm{C}$ implementation of our $\lambda \mathrm{DP} / \mathrm{RD}$ algorithm to obtain the $\lambda \mathrm{DP}^{\mathrm{BM}}$ for the $40 \mathrm{CNs}$. All simulations were run on a $2 \mathrm{x}$ Intel Pentium 2-2.6Ghz with $1.8 \mathrm{~GB}$ of RAM, running Fedora Core 6.

\section{A. The Effects of $k$ on Reliability}

We ran $\lambda \mathrm{DP} / \mathrm{RD}$ algorithm on all the 40 networks with increasing $k=1,2, \ldots$; this simulation is used to show the correctness of Theorems 1 and 2. The results for each network are consistent with the theorems; Table II shows the results when the algorithm was executed on one of the $\mathrm{CN} 3 \mathrm{~s}$ that had $\mathrm{D}_{\min }=23$.

TABLE II

RESULTS OBTAINED FROM $\lambda$ DP/RD ALGORITHM WITH ONE CN3

\begin{tabular}{|c|c|c|}
\hline $\boldsymbol{k}$ & Delay & Reliability \\
\hline 1 to 12 & 40 & 0.825406 \\
\hline 13 to 16 & 40 & 0.816747 \\
\hline 17 to 22 & 40 & 0.804262 \\
\hline 23 to 32 & 31 & 0.786859 \\
\hline 33 to 34 & 31 & 0.75805 \\
\hline 35 to 41 & 31 & 0.71994 \\
\hline 42 & 31 & 0.667086 \\
\hline
\end{tabular}

Consistent with Theorem 1, when $k=1$, the algorithm produced the $\lambda \mathrm{DP}$ with the highest reliability. It remained as the $\lambda \mathrm{DP}^{\mathrm{BM}}$ until $k=23$ at which $\lambda \mathrm{DP}^{\mathrm{NM}}$ had a lower delay; this $\lambda \mathrm{DP}^{\mathrm{NM}}$ became the new $\lambda \mathrm{DP}^{\mathrm{BM}}$ since its reliability $\geq \mathrm{R}=0.7$. For $k=42, \quad \lambda \mathrm{DP}^{\mathrm{NM}}$ has reliability $0.667086<\mathrm{R}$. Thus, the algorithm outputs as the solution with the smallest delay the $\lambda \mathrm{DP}^{\mathrm{BM}}$ generated when $k=23$. Note that for this $\mathrm{CN}$, the delay $\delta\left(\lambda \mathrm{DP}^{\mathrm{NM}}\right)$ decreased as $k$ increased; however, this may not always be true. Therefore, we need to increase $k$ sequentially to evaluate every $\lambda \mathrm{DP}^{\mathrm{NM}}$ using Eqs. (7) and (8) until a value of $k$ produces a $\lambda D P^{\mathrm{NM}}$ with $\rho\left(\lambda \mathrm{DP}^{\mathrm{NM}}\right)<\mathrm{R}$ before deciding on the $\lambda \mathrm{DP}^{\mathrm{BM}}$. To validate Theorem 2 , we checked that each feasible solution has $\mathrm{D}_{\min } \leq \mathrm{W}\left(\lambda \mathrm{DP}^{k}, \eta_{k}\right) \leq(1+2 k) \mathrm{D}_{\min }$.

\section{B. The Accuracy of the Algorithm}

We used our algorithm to generate $\lambda \mathrm{DP}^{\mathrm{BM}}$ and computed its delay $\left(\mathrm{D}_{\text {ours }}\right)$ and reliability $\left(\mathrm{R}_{\text {ours }}\right)$ as shown in Table III. To evaluate the optimality of our algorithm, we compared $\mathrm{D}_{\text {ours }}$ and $\mathrm{R}_{\text {ours }}$ with $\mathrm{D}_{\mathrm{BF}}$ and $\mathrm{R}_{\mathrm{BF}}$, respectively, which were generated using an exponential time brute force (BF) algorithm (described in Section III). Note that $D_{\text {ours }}\left(R_{\text {ours }}\right)$ always satisfies the delay bound $\mathrm{D}_{\text {bound }}=(1+k) \mathrm{D}_{\text {min }}$ (reliability constraint $\mathrm{R}$ ) for each $\mathrm{CN}$.

We consider four possible comparison results: (i) $\mathrm{D}_{\text {ours }}=\mathrm{D}_{\mathrm{BF}}$ and $\mathrm{R}_{\text {ours }}=\mathrm{R}_{\mathrm{BF}}$, (ii) $\mathrm{D}_{\text {ours }}=\mathrm{D}_{\mathrm{BF}}$ and $\mathrm{R}_{\text {ours }}<\mathrm{R}_{\mathrm{BF}}$, (iii) $D_{\text {ours }}>D_{B F}$ and $R_{\text {ours }} \geq R_{B F}$, and (iv) $D_{\text {ours }}>D_{B F}$ and $R_{\text {ours }}<R_{B F}$. The columns " $\% \mathrm{D}_{\text {ours }}$ " and " $\% \mathrm{R}_{\text {ours }}$ " in Table III show the percentage differences between $D_{\text {ours }}$ and $R_{\text {ours }}$ against $D_{B F}$ 
and $\mathrm{R}_{\mathrm{BF}}$ respectively. As shown in column 8 of Table III our algorithm is optimal $25 \%$ of the time (marked with (i)) both in terms of delay and reliability, and $47.5 \%$ of the time it generates the same delay as BF but with, on average, $2.6 \%$ less reliable (a negative value marked with (ii)). Note that $5 \%$ of the time our algorithm produced results in category (iii) (marked with (iii)), where there is a tradeoff of a higher delay (average 9.59\%) for higher reliability (average $1.95 \%$ ). Even though $22.5 \%$ of the time our approach produced results in category (iv) (marked with (iv)), $\mathrm{D}_{\text {ours }}$ is at most $9.76 \%$ higher than $\mathrm{D}_{\mathrm{BF}}$ and $\mathrm{R}_{\text {ours }}$ is at most $2.38 \%$ lower than $\mathrm{R}_{\mathrm{BF}}$. Our $\lambda \mathrm{DP} / \mathrm{RD}$ algorithm and the $\mathrm{BF}$ approach took on average 1.2 seconds and 191 seconds respectively to generate the $\lambda \mathrm{DP}^{\mathrm{BM}}$ of each $\mathrm{CN}$.

TABLE III

COMPARISON BETWEEN $\lambda$ DP/RD AND BF RESULTS

\begin{tabular}{|c|c|c|c|c|c|c|c|c|}
\hline & & & \multicolumn{3}{|c|}{ Delay } & \multicolumn{3}{|c|}{ Reliability } \\
\hline $\mathbf{C N}$ & $\boldsymbol{k}_{\mathrm{opt}}$ & $\boldsymbol{k}_{\max }$ & $\mathbf{D}_{\mathrm{BF}}$ & $\% D_{\text {ours }}$ & $D_{\text {bound }}$ & $\mathbf{R}_{\mathrm{BF}}$ & $\% \mathbf{R}_{\text {ours }}$ & $\% R_{\text {ours }}^{\prime}$ \\
\hline \multicolumn{9}{|c|}{ CN1, $R=0.1$} \\
\hline 1 & 49 & 66 & 40 & 0.00 & 1550 & 0.1038 & -3.62 (ii) & -3.62 \\
\hline 2 & 1 & 59 & 39 & 0.00 & 62 & 0.1025 & 0.00 (i) & 0.00 \\
\hline 3 & 23 & 56 & 39 & 0.00 & 792 & 0.1015 & 0.00 (i) & 0.00 \\
\hline 4 & 1 & 8 & 40 & 0.00 & 60 & 0.1035 & -1.28 (ii) & -1.28 \\
\hline 5 & 9 & 15 & 41 & 0.00 & 155 & 0.1002 & 0.00 (i) & 0.00 \\
\hline 6 & 3 & 47 & 45 & 0.00 & 124 & 0.1001 & 0.00 (i) & 0.00 \\
\hline 7 & 17 & 37 & 40 & 2.50 & 512 & 0.1083 & -0.51 (iv) & -0.51 \\
\hline 8 & 12 & 59 & 40 & 2.50 & 403 & 0.1096 & -1.95 (iv) & -1.95 \\
\hline 9 & 10 & 77 & 39 & 2.56 & 341 & 0.1130 & -0.22 (iv) & -0.22 \\
\hline 10 & 25 & 53 & 41 & 9.76 & 806 & 0.1052 & -0.20 (iv) & -0.21 \\
\hline \multicolumn{9}{|c|}{ CN2, R=0.5 } \\
\hline 1 & 12 & 35 & 32 & 12.50 & 286 & 0.5082 & 2.67 (iii) & -1.22 \\
\hline 2 & 2 & 36 & 31 & 0.00 & 66 & 0.5111 & 0.00 (i) & 0.00 \\
\hline 3 & 1 & 25 & 32 & 0.00 & 42 & 0.5217 & 0.00 (i) & 0.00 \\
\hline 4 & 35 & 37 & 32 & 3.13 & 805 & 0.5330 & -4.10 (iv) & -4.10 \\
\hline 5 & 27 & 47 & 32 & 0.00 & 532 & 0.5328 & -1.80 (ii) & -1.80 \\
\hline 6 & 1 & 42 & 31 & 0.00 & 46 & 0.5629 & 0.00 (i) & 0.00 \\
\hline 7 & 37 & 49 & 30 & 6.67 & 816 & 0.5253 & 1.23 (iii) & -0.66 \\
\hline 8 & 51 & 63 & 32 & 0.00 & 1040 & 0.5423 & -5.77 (ii) & -5.77 \\
\hline 9 & 1 & 36 & 32 & 0.00 & 44 & 0.5270 & 0.00 (i) & 0.00 \\
\hline 10 & 3 & 60 & 38 & 0.00 & 80 & 0.5251 & 0.00 (i) & 0.00 \\
\hline \multicolumn{9}{|c|}{$\mathrm{CN} 3, \mathrm{R}=0.7$} \\
\hline 1 & 23 & 42 & 33 & 0.00 & 552 & 0.8078 & -2.60 (ii) & -2.60 \\
\hline 2 & 47 & 52 & 31 & 0.00 & 912 & 0.7398 & -1.84 (ii) & -1.84 \\
\hline 3 & 17 & 27 & 31 & 3.23 & 374 & 0.7568 & -2.28 (iv) & -2.28 \\
\hline 4 & 1 & 17 & 31 & 0.00 & 40 & 0.8277 & -3.97 (ii) & -3.97 \\
\hline 5 & 36 & 55 & 34 & 2.94 & 740 & 0.7710 & -2.38 (iv) & -2.38 \\
\hline 6 & 1 & 17 & 32 & 0.00 & 34 & 0.7440 & -1.17 (ii) & -1.17 \\
\hline 7 & 19 & 65 & 30 & 0.00 & 420 & 0.8277 & -5.38 (ii) & -5.38 \\
\hline 8 & 22 & 43 & 29 & 0.00 & 460 & 0.7477 & 0.00 (i) & 0.00 \\
\hline 9 & 5 & 37 & 33 & 0.00 & 54 & 0.7507 & -0.10 (ii) & 0.10 \\
\hline 10 & 1 & 19 & 34 & 0.00 & 50 & 0.7472 & -3.67 (ii) & -3.67 \\
\hline \multicolumn{9}{|c|}{$\mathrm{CN} 4, \mathrm{R}=0.95$} \\
\hline 1 & 42 & 43 & 32 & 0.00 & 1032 & 0.9999 & -3.89 (ii) & -3.89 \\
\hline 2 & 44 & 68 & 29 & 0.00 & 1035 & 0.9999 & -1.87 (ii) & -1.87 \\
\hline 3 & 75 & 84 & 28 & 3.57 & 1520 & 0.9999 & -1.12 (iv) & -1.12 \\
\hline 4 & 55 & 59 & 29 & 0.00 & 1092 & 0.9999 & -1.20 (ii) & -1.20 \\
\hline 5 & 31 & 57 & 28 & 0.00 & 640 & 0.9997 & -0.57 (ii) & -0.57 \\
\hline 6 & 32 & 48 & 32 & 0.00 & 759 & 0.9999 & -0.85 (ii) & -0.85 \\
\hline 7 & 21 & 46 & 29 & 3.45 & 462 & 0.9999 & -0.01 (iv) & -0.01 \\
\hline 8 & 51 & 65 & 32 & 0.00 & 987 & 0.9999 & -0.78 (ii) & -0.78 \\
\hline 9 & 25 & 36 & 29 & 0.00 & 520 & 0.9999 & -0.30 (ii) & -0.30 \\
\hline 10 & 19 & 57 & 29 & 0.00 & 420 & 0.9999 & -0.03 (ii) & $\begin{array}{l}-0.03 \\
\end{array}$ \\
\hline
\end{tabular}

To see the optimality of our algorithm in terms of the reliability of the $\lambda \mathrm{DP}^{\mathrm{BM}}$, we compared its results with those generated by the $\mathrm{BF}$ approach while setting $\mathrm{D}_{\mathrm{BF}}=\mathrm{D}_{\text {ours }}$. The column $\% \mathrm{R}_{\text {ours }}$ in Table III shows the percentage differences between the reliability obtained by our algorithm against that obtained by BF. As indicated earlier (shown in column 8 in Table III), $25 \%$ of the time, our algorithm generates optimal reliability $(0 \%$ in the column). Even though $75 \%$ of the time our approach does not produce the $\lambda \mathrm{DP}^{\mathrm{BM}}$ with optimal reliability, $\mathrm{R}_{\text {ours }}$ is at most only $-5.77 \%$ off the optimal result, while using only $0.79 \%$ of the $\mathrm{CPU}$ time required by the optimal BF approach.
Table III shows $k_{\max }$ and $k_{\text {opt }}$ that denote the value of the smallest $k$ for which the algorithm results in $\rho\left(\lambda \mathrm{DP}^{\mathrm{NM}}\right)<\mathrm{R}$ (thus it outputs $\lambda \mathrm{DP}^{\mathrm{BM}}$ ) and the value of $k$ when $\rho\left(\lambda \mathrm{DP}^{\mathrm{BM}}\right)=\max \left(\left\{\rho\left(\lambda \mathrm{DP}_{\sigma}^{r d}\right)\right\}\right)$, respectively. As shown in column $3, k_{\max }<85$, i.e., our algorithm in Fig. 3 iterates the repeat loop at most 84 times for generating each $\lambda \mathrm{DP}^{\mathrm{BM}}$.

\section{CONCLUSION}

We have addressed an important $\lambda \mathrm{DP} / \mathrm{RD}$ problem to generate a $\lambda \mathrm{DP}$ with minimum delay while meeting a reliability constraint, R. An approximate Lagrangerelaxation algorithm has been presented to solve the problem. Our simulations on forty randomly generated CNs with random edge reliabilities and delays show that our polynomial time method is able to generate $\lambda \mathrm{DP} / \mathrm{RD}$ with delay and reliability values comparable to those generated using the optimal but time-expensive brute force approach.

We are investigating a method to bound the value of $k$ to further reduce the complexity of our approach. We also plan to use some alternative heuristic algorithms for the problem.

\section{REFERENCES}

[1] R. C. Loh, S. Soh, M. Lazarescu, and S. Rai, "A Greedy Technique for Finding the Most Reliable Edge-disjoint-path-set in a Network," Proc. IEEE PRDC, Taiwan, pp. 216-223, 2008.

[2] R. C. Loh, S. Soh, and M. Lazarescu, "An Approach to Find Maximal Disjoint Paths with Reliability and Delay Constraints," Proc. IEEE AINA, Bradford, UK, pp. 959-964, 2009.

[3] Y. Guo, F. Kuipers, and P. Van Mieghem, "Link-disjoint paths for reliable QoS routing," Int'l J. Comm. Sys., vol. 16, pp. 779-798, 2003.

[4] X. Huang and Y. Fang, "Multiconstrained QoS multipath routing in wireless sensor networks," Wireless Networks, vol. 14, pp. 465-478, 2008.

[5] M. Razzaque, A. Alam, M. M. Mamun-Or-Rashid, M. Hong, and C. Seon, "Multi-Constrained QoS Geographic Routing for Heterogeneous Traffic in Sensor Networks," 5th IEEE CCNC, pp. 157-162, 2008.

[6] R. C. Loh, S. Soh, and M. Lazarescu, "Finding the most reliable edge-disjoint-path-set in a communication network," Proc. PEECS, Perth, Australia, pp. 121-126, 2007.

[7] A. Agarwal and B. Jain, "Routing reliability analysis of segmented backup paths in mobile ad hoc networks," ICPWC, pp. 52-56, 2005.

[8] C. Peng and H. Shen, "A New Approximation Algorithm for Computing 2-Restricted Disjoint Paths," IEICE Trans. Info. and Sys., vol. 90, pp. 465-472, 2007.

[9] P. Papadimitratos, Z. J. Haas, and E. G. Sirer, "Path set selection in mobile ad hoc networks," Proc. ACM MobiHoc, Lausanne, Switzerland, pp. 1-11, 2002.

[10] A. K. Andreas, J. C. Smith, and S. Kucukyavuz, "Branch-and-Priceand-Cut Algorithms for Solving the Reliable h-Paths Problem," October 9, 2007.

[11] A. K. Andreas and J. C. Smith, "Exact Algorithms for Robust kPath Routing Problems," Procs. of GO, pp. 1-6, 2005.

[12] V. Guruswami, S. Khanna, R. Rajaraman, B. Shepherd, and M. Yannakakis, "Near-optimal hardness results and approximation algorithms for edge-disjoint paths and related problems," J. Comp. and Sys. Sci., vol. 67, pp. 473-496, 2003.

[13] A. Orda and A. Sprintson, "Efficient algorithms for computing disjoint QoS paths," Proc. IEEE INFOCOM, Hong Kong, vol. 1, pp. 727-738, 2004.

[14] G. Xue, W. Zhang, J. Tang, and K. Thulasiraman, "Polynomial time approximation algorithms for multi-constrained QoS routing," IEEE Trans. Networking, vol. 16, pp. 656-669, 2008.

[15] S. Soh, S.Rai, "Telecommunication Network Reliability," Encyclopedia of Life Support Systems, UNESCO/Eolss Publishers, Oxford, U.K., 2007.

[16] A. Medina, A. Lakhina, I. Matta, and J. Byers, "BRITE: Universal Topology Generation from a User's Perspective," Proc. IEEE MASCOTS, Ohio, USA, pp. 346-353, 2001. 